Document downloaded from:

http://hdl.handle.net/10251/61313

This paper must be cited as:

Juan, M.; Calatrava, J. (2011). An Augmented Reality system for the treatment of phobia to small animals viewed via an optical see-through HMD. Comparison with a similar system viewed via a video see-through. International Journal of Human-Computer Interaction. 27(5):436-449. doi:10.1080/10447318.2011.552059.

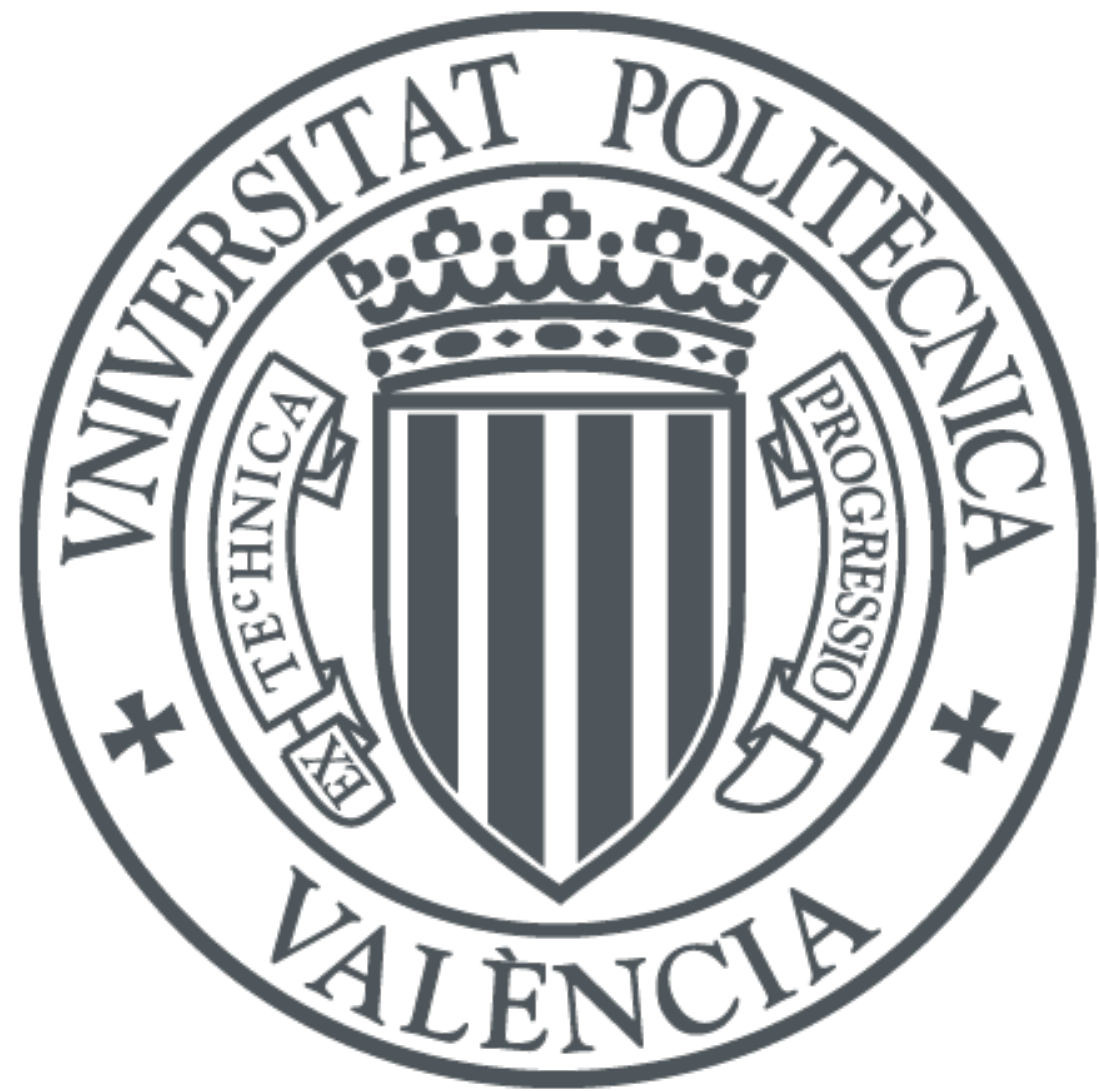

The final publication is available at

http://dx.doi.org/10.1080/10447318.2011.552059

Copyright Taylor \& Francis

Additional Information 


\title{
An Augmented Reality system for the treatment of phobia to small animals viewed via an optical see-through HMD. Comparison with a similar system viewed via a video see-through HMD
}

\author{
M. Carmen Juan ${ }^{1 *}$, Jérôme Calatrava ${ }^{2}$ \\ ${ }^{1}$ Instituto Universitario de Automática e Informática Industrial, Universitat Politècnica de València, \\ Camino de Vera, s/n. 46022 Valencia (Spain) \\ ${ }^{2}$ Université Paul Sabatier (Toulouse III), Toulouse (France)
}

\begin{abstract}
This paper presents an optical see-through (OST) Augmented Reality (AR) system for the treatment of phobia to small animals. The technical characteristics of the OST system are described, and a comparative study of the sense of presence and anxiety in a non-phobic population (24 participants) using the OST and an equivalent video see-though (VST) system is presented. The results indicate that if all participants are analyzed, the VST system induces greater sense of presence than the OST system. If the participants who had more fear are analyzed, the two systems induce a similar sense of presence. For the anxiety level, the two systems provoke similar and significant anxiety during the experiment.
\end{abstract}

Keywords: Augmented Reality, Optical see-through, virtual therapy, phobia to small animals, presence, anxiety

\section{Introduction}

The term Augmented Reality (AR) is used to describe systems that blend computer-generated virtual objects with real environments. In this paper, we present an AR optical see-through (OST) system for the treatment of phobia to small animals. People who suffer from arachnophobia or other types of phobia to small animals become anxious when they are in a situation where these animals can appear. They suffer an unrealistic and excessive fear that makes life miserable since they are always frightened of seeing the animal they fear. Our system is not the first AR system that uses OST HMD, but this is the first time OST has been developed for the treatment of phobia to small animals. Several AR systems that use OST HMD for different purposes have been reported in the literature: collaborative applications for architectural design (Grasset et al. 2001); showing hidden structures of buildings (Ivanovic \& Plate, 2002); training and assisting in maintaining equipment in an industrial context (Schwald \& Laval, 2003); gaming (Ozbek et al., 2004); and disaster management (Leebmann, 2006).

Phobias of cockroaches and spiders have already been treated with AR using a video see-through (VST) system (Juan et al., 2005; Botella et al., 2005). The work presented here in this paper is the OST version of the same VST system. The work by Juan et al. (2005) \& Botella et al. (2005) demonstrated that, with a single one-hour session, patients significantly reduced their fear and avoidance. Initially, the VST system was tested in a case study (Botella et al., 2005), and then it was tested on nine patients suffering from phobia of small animals (Juan et al., 2005). An invisible marker version of the VST system was also presented (Juan et al., 2006a). For the treatment of acrophobia, Juan et al. (2006b) proposed the use of immersive photography in an AR system for the treatment of this phobia. In that system, forty-one participants without acrophobia walked around at the top of a staircase in both a real environment and in an immersive photography environment. The users' scores for presence in the immersive photography environment were very high. The results indicated that the acrophobic context could be useful for the treatment of acrophobia. However, statistically significant differences were found between the real and the immersive photography environments. 
Phobia to small animals has also been treated using Virtual Reality (VR). Carlin, Hoffman \& Weghorst (1997) used immersive VR for exposure therapy. The first experiment was carried out at the U.W. Human Interface Technology laboratory (HITLab) (www.hitl.washington.edu/ projects/exposure). The first patient treated with that system needed twelve one-hour VR therapy sessions. In another work, Renaud, Bouchard \& Proulx (2002) compared tracking behaviour with a virtual spider and a neutral target in both fearful and non-fearful subjects. Garcia et al. (2002) also explored the effectiveness of VR exposure therapy for the treatment of spider phobia. They compared a VR treatment condition vs. a waiting list condition (participants waiting for treatment, but without treatment) in a between group design with 23 participants. Participants in the VR treatment group received an average of four one-hour exposure therapy sessions. VR exposure was effective in treating spider phobia compared to a control condition as measured with a "fear of spiders" questionnaire, a behavioural avoidance test, and severity ratings made by the clinician and an independent assessor. A total of $83 \%$ of the patients in the VR treatment group showed clinically significant improvement compared with $0 \%$ in the waiting list group, and no patients dropped out. Hoffman et al. (2003) explored whether giving patients the illusion of physically touching a virtual spider increased treatment effectiveness. Eight clinically phobic students and 28 non-clinically phobic students participated in that study. The participants were randomly assigned to one of three groups (no treatment, VR with no tactile cues, or VR with tactile cues). The participants in the two VR treatment groups received three one-hour exposure therapy sessions. The tactile augmentation group showed the greatest progress in behavioral measures. Botella et al. (2004) presented a telepsychology system that uses VR to treat phobia to small animals (cockroaches, spiders and rats) where patients follow the treatment in their own home. In that system, a typical kitchen was modelled as the environment. The system had different levels at which one or more small animals could appear. The animals randomly appeared when the user opened the door of a cupboard.

\section{Optical see-through system}

The hardware for the system consists of a camera for capturing the real world in order to determine exactly where the virtual elements will have to be drawn. To do this, we used an infrared (IR) camera, but a color camera could also be used. The IR bullet camera that we used is shown in Figure 1.a. It has a $715 \mathrm{~nm}$ IR filter and comes in a lipstick-sized tube that is 2.5 inches long, with a diameter of 0.8125 inches. The diagonal FOV of the camera is 92 degrees. The image sensor is 1/3" CCD with a maximum frame rate of $30 \mathrm{fps}$ in several image formats, among them $640 \times 480$. A LitEye500 monocular OST HMD was used as the visualization hardware (Fig 1.b). The resolution of the HMD enables $800 \times 600$ with a FOV of 28 degrees. Using a screen, the person in charge of the tests could see the same scene as the participant on the HMD. The IR camera was firmly attached to the HMD (Figure 1.c).

The software that we used was the ARToolKit library (Kato \& Billinghurst, 1999) with Virtual Reality Modeling Language (VRML). ARToolKit is an open-source vision-tracking library that uses computer vision techniques to obtain the position and orientation of the camera with respect to a marker. Virtual elements are drawn on these markers. The required elements for the application are: a USB or FireWire camera, and a marker. Markers are white squares with a black border inside of which are symbols or letters. The three-dimensional models of the virtual elements were designed using Autodesk 3D Studio Max. These models were exported to VRML format and edited with VRMLPad. Textures were created in Adobe Photoshop. The graphical user interface was created using the OpenGL Utility ToolKit (GLUT)-based user interface library (GLUI). Sound support was provided by the OpenAL sound library. 


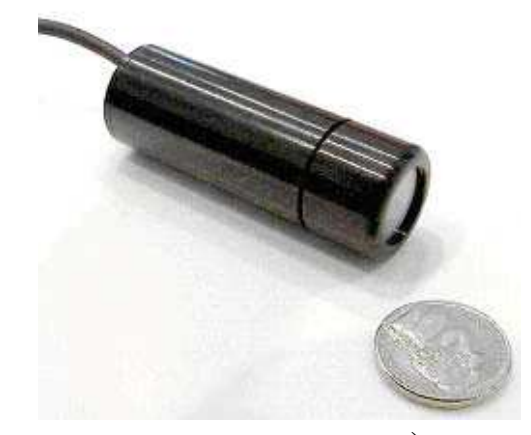

a)

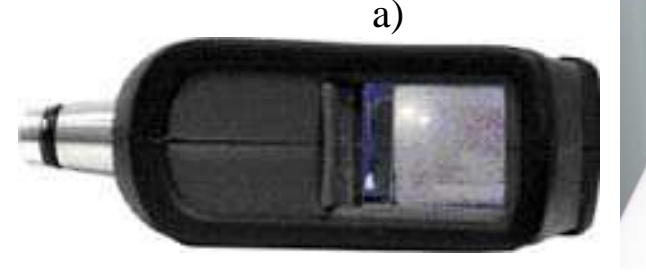

b)

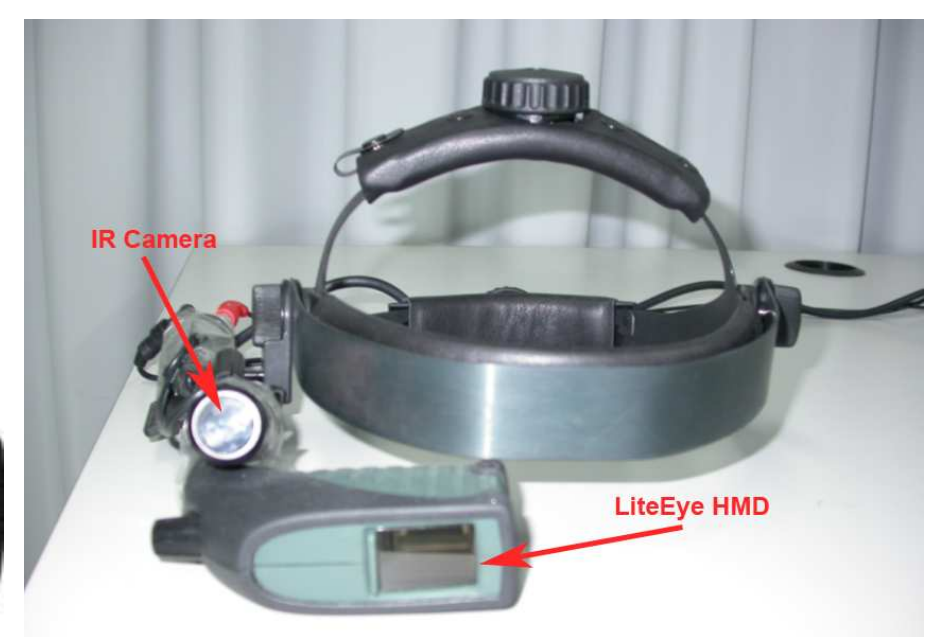

c)

Figure 1. a) The IR bullet camera used b) The LiteEye-500 monocular OST HMD. c) Capture and visualization system (IR camera and the LiteEye HMD)

Since the system is OST, the calibration process is much more complicated than in a VST system because the human subject is an inseparable part of the eye-display system (Owen et al., 2004). Several procedures for the calibration of OST system have already been proposed (e.g., Azuma \& Bishop, 1994; Fuhrmann, Schmalstieg \& Purgathofer, 1999; Grasset et al., 2001; Owen el al., 2004; Genc et al., 2002). Kato and Billinghurst (1999) proposed a calibration method for optical see-through HMD based on marker tracking. We have adapted this method for the calibration of the monocular OST HMD. In an OST HMD, a ray from a physical object reaches the focal point of the eye through the HMD screen. Then, a 3D position that is represented in the eye coordinates whose origin is the focal point of the eye can be projected on the HMD screen coordinates by the perspective projection model. This assumes that the $\mathrm{Z}$ axis perpendicularly crosses the HMD screen and the $\mathrm{X}$ and $\mathrm{Y}$ axes are parallel to the $\mathrm{X}$ and $\mathrm{Y}$ axes of the HMD screen coordinate frame, respectively. Figure 2 shows the coordinate frames in the calibration procedure.

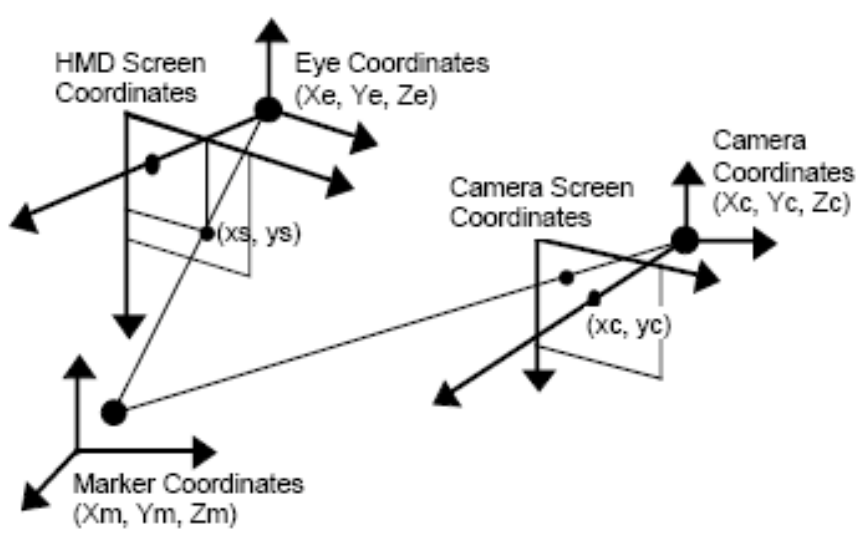

Figure 2. Coordinate frames in the calibration procedure

Pose estimation of a marker is done by calculating the transformation matrix from marker coordinates to camera coordinates, $\mathrm{T}_{\mathrm{cm}}$. The perspective projection matrix $\mathrm{P}$ is required in this procedure. Camera calibration finds the perspective projection matrix $\mathrm{P}$ that represents the relationship between the camera coordinates and the camera screen coordinates. In order to display virtual objects 
on a HMD screen as if they are on the marker, the relationship between the marker coordinates and the HMD screen coordinates is required. Therefore, the relationship between the HMD screen coordinates and eye coordinates is required and represented by the perspective projection. The relationship between the camera coordinates and eye coordinates is represented by rotation and translation transformations. Equation 1 shows these relationships, where $Q_{s e}$ represents the perspective transformation matrix, and $\mathrm{T}_{\mathrm{ec}}$ represents the rotation and translation matrix.

$$
\left[\begin{array}{c}
i x_{S} \\
i y_{S} \\
i \\
1
\end{array}\right]=Q_{s e}\left[\begin{array}{c}
X_{e} \\
Y_{e} \\
Z_{e} \\
1
\end{array}\right]=Q_{s e} T_{e c}\left[\begin{array}{c}
X_{C} \\
Y_{C} \\
Z_{C} \\
1
\end{array}\right]=Q_{s e} T_{e c} T_{c m}\left[\begin{array}{c}
X_{m} \\
Y_{m} \\
Z_{m} \\
1
\end{array}\right] \text { (equation 1) }
$$

The OST version uses the same four VRML models as the VST system. We have three different spiders and one cockroach. For each type of animal, three models were designed: a non-moving, a moving, and a dead animal. To obtain a result as real as possible, the moving cockroach was animated with moving legs and moving tentacles, and the spiders move their legs. When the animals are killed, the system produces a sound similar to that of a real animal being killed. The system includes two sounds: a squirting sound similar to the sound of a real can of insecticide; and a squishing sound similar to that of a real cockroach or spider being crushed.

With regard to the OST calibration process, it has to be complete before the system is run. A calibration process was performed and a default file was created. Initially, the system first loads this default calibration file and it normally works properly; however, for better results, the calibration process should be performed for each user (differences in visual perception among users).

The IR camera captures the scene of the real world. The entry of the IR camera is treated by the system and the position and orientation of the IR camera with respect to the marker can be established using ARToolKit. Then, the system simply draws the animals in the right place because the user is seeing the real world.

Since the functionality of the system is the same as the marker system (Juan et al., 2005), in this paper, we only comment on its functionalities briefly. The user can select the number of animals to appear: one animal, increase/decrease by three animals, increase/decrease by 20 animals. In addition, the size of the animal/s can be increased/reduced and they can move or stop. It is also possible to kill the animals using two different elements. When this occurs, the system plays a sound that is related to the tool being used and one or more dead animals appear. If the spider system is used, there are three types of spiders to choose from.

\section{Results}

The aim of this study was to test which AR system (VST or OST) induces more sense of presence and provokes more anxiety in users. To do this, we compared subjective presence measurements that were collected after exposure to the VST AR system and the OST AR system. To determine the level of anxiety, the participants were asked to rate their anxiety level at three different moments.

\section{Participants}

Twenty-five participants took part in the study (12 males, 13 females). The average age of the 24 participants was 32.63 years (S.D., 6.64). The participants did not receive any compensation for their time. All the participants filled out the "fear and avoidance of cockroaches and spiders" questionnaires (which were adapted from the Szymanski \& O'Donohue (1995) questionnaire). These questionnaires were analyzed. If participants had more phobia to spiders than cockroaches, they were exposed to the 
spider AR system; otherwise, they were exposed to the cockroach AR system. Our initial idea was to divide the participants of the two groups into two subgroups; participants having a fear score of more than 97 (the clinical group, i.e., phobic people), and participants with a fear score of less than or equal to 97 (the normal group, i.e., non-phobic people). This value (97) is normally used as the cut-off value for distinguishing phobic people from non-phobic people (e.g. in Garcia et al., 2002). Only one participant had a fear score of more than 97 . Her fear score was 108, and she was excluded from the study. The participants' fear scores were analyzed to determine the value that separated the participants into the two subgroups, which, in this case, was 50. The participants were then divided into two groups: "low fear subjects", with a fear score of less than or equal to 50; and "high fear" subjects, with a fear score of more than 50. Five of the participants were not asked about their anxiety levels during the experiment. As a result, for the anxiety level, there were nineteen participants (twelve for the low fear group and seven for the high fear group). The participants read and signed a consent form accepting the exposure that they were going to receive, allowing us to videotape the sessions, and allowing us to use their data in our research.

\section{Procedure}

The participants were counterbalanced and randomly assigned to one of two conditions:

a) Participants who used the VST AR system first and then the OST AR system.

b) Participants who used the OST AR system first and then the VST AR system.

The protocol was the following. Before the participants were exposed to the OST or the VST AR systems, they received instructions about how to interact with the system during the experiment. After using each system (OST or VST), the participants were asked to fill out an adapted Slater et al. questionnaire (Slater, Usoh \& Steed, 1994) (SUS). Hence, the participants filled out this questionnaire twice. The 6 questions related to the sense of presence were as follows. The scoring was on a scale of 1-7. Q1-Please rate your sense of being in a room where there are cockroaches/spiders (where 7 represents your normal experience of being in a place); Q2-To what extent were there times during the experiment when the cockroaches/spiders were real for you?; Q3-When you think back to your experience, do you think of cockroaches/spiders more as images that you saw (a movie, a picture), or more as cockroaches/spiders that were in the same room as you were?; Q4-During the experiment, which was strongest on the whole: your sense of being in the room where there were cockroaches/spiders, or your sense of being in a room without cockroaches/spiders?; Q5-Think about your memory of being in "a room where there were cockroaches/spiders". How similar is this memory to your memories of other places where there were these animals?; Q6-During the experiment, did you often think that you were actually in a room where there were cockroaches/spiders?

For the level of anxiety, the participants were asked to rate their anxiety level (scores from $0=$ not anxious at all, to $10=$ very anxious) at three different moments. These moments were: P1-Before starting the experiment; P2-When participants put their hand on the table and animals crossed over it; and P3- At the end of the exposure. In this experiment, the anxiety level was only asked for the first time that a participant used one of the two systems: if a participant used the OST AR system first, he/she was asked for his/her anxiety level during this experiment and was not asked for the anxiety level when he/she used the VST AR system second.

\section{Data analysis}

For the sense of presence, Table 1 shows the results related to presence measures. The significance level was set to 0.05 in all tests. All of the participants were considered. Paired t-tests were applied to the scores given to all of the questions. The Mean column uses the mean score across the six questions. The remaining columns show the mean results for the individual questions. The results from questions Q1-Q5 show significant statistical differences between the two systems when tests were applied. These results indicate that the participants considered the VST system to be clearly different and induced more sense of presence than the OST system. The results from Q6 show no significant statistical 
differences. For this question, participants had a similar sense of presence using the two systems. In order to determine whether using one of the two systems first has some effect on the presence measurement for the second system, the sample was divided into two groups (the participants who used the OST system first and the participants who used the VST system first). Student t tests assuming equal variances were applied to the scores given to all of the questions. In this analysis, no significant statistical differences were found. This indicates that the order in which the participants used the systems did not influence their presence scores. In order to determine if participants who had high fear showed the same results as all the population, a new paired t-test analyzing only these participants $(\mathrm{N}=7)$ was performed (see Table 2). The results from all of the questions show no significant statistical differences between the two systems. This implies that the two systems induced a similar sense of presence in this subgroup. To sum up, if all the participants are analyzed, the VST system induces greater sense of presence than the OST system and the order of exposure did not change the results. If the participants who had high fear are analyzed, the two systems induce a similar sense of presence.

For anxiety levels, we distinguished between the two groups, subjects having less fear $(\mathrm{N}=12)$ and subjects with more fear $(\mathrm{N}=7)$. The anxiety values were collected during the exposure at moments $\mathrm{P} 1$ P3. Table 3 shows Student t tests assuming equal variances for the scores given in response to these questions for the two systems for all the participants. The results from Table 3 show that there is no significant difference in the anxiety level using the two systems. This implies that the two systems produce a similar level of anxiety in these steps. In order to determine whether the participants with more/less fear report more anxiety, the scores of the group of participants with more fear/less fear were analyzed, and Student $t$ tests assuming equal variances were applied to the scores given to all questions (see Tables 4-5). We compared the anxiety level at the moment before starting the experiment with the anxiety level during the different stages of the experiment using the two systems, and we also analyzed the scores of participants who had more/less fear. Paired t-tests are shown in Tables 6-11. The results indicate that there is a significant difference between the initial anxiety and the anxiety felt in step 2 for all conditions. That is, the two systems produce significant anxiety in participants. To sum up, the two systems produced similar and significant anxiety during the experiment. Finally, several images of the running OST system are shown in Figures 3-6.

\begin{tabular}{|l|l|l|l|l|l|l|l|}
\hline & \multicolumn{1}{|c|}{ Mean } & \multicolumn{1}{|c|}{ Q1 } & \multicolumn{1}{|c|}{ Q2 } & \multicolumn{1}{|c|}{ Q3 } & \multicolumn{1}{c|}{ Q4 } & \multicolumn{1}{c|}{ Q5 } & Q6 \\
\hline OST & $4.44 \pm 1.79$ & $4.50 \pm 1.69$ & $4.58 \pm 1.74$ & $4.38 \pm 1.91$ & $4.50 \pm 1.87$ & $4.13 \pm 1.78$ & $4.54 \pm 1.89$ \\
\hline VST & $5.39 \pm 1.59$ & $5.71 \pm 1.30$ & $5.50 \pm 1.35$ & $5.34 \pm 1.64$ & $5.54 \pm 1.56$ & $5.17 \pm 1.81$ & $5.04 \pm 1.85$ \\
\hline $\mathrm{t}$ & & $-4.61^{* *}$ & $-4.61 * *$ & $-3.92^{* *}$ & $-3.57 * *$ & $-3.30^{* *}$ & -1.57 \\
\hline $\mathrm{p}$ & & $<0.001^{* *}$ & $<0.001^{* *}$ & $<0.001^{* *}$ & $0.002^{* *}$ & $0.003^{* *}$ & 0.130 \\
\hline
\end{tabular}

Table 1. Mean and standard deviation of presence scores, d.f. 23, ' $* *$ ' indicates significant differences.

\begin{tabular}{|l|l|l|l|l|l|l|}
\hline & \multicolumn{1}{|c|}{ Q1 } & \multicolumn{1}{|c|}{ Q2 } & \multicolumn{1}{|c|}{ Q3 } & \multicolumn{1}{|c|}{ Q4 } & \multicolumn{1}{c|}{ Q5 } & \multicolumn{1}{c|}{ Q6 } \\
\hline OST & $5.57 \pm 1.27$ & $5.57 \pm 1.81$ & $5.00 \pm 2.08$ & $5.71 \pm 1.11$ & $5.14 \pm 1.35$ & $5.29 \pm 1.98$ \\
\hline VST & $6.14 \pm 1.21$ & $6.00 \pm 1.53$ & $5.71 \pm 1.80$ & $6.29 \pm 1.50$ & $5.86 \pm 1.68$ & $5.71 \pm 1.98$ \\
\hline $\mathrm{t}$ & -1.92 & -1.44 & -1.18 & -1.00 & -1.26 & -0.81 \\
\hline $\mathrm{p}$ & 0.103 & 0.200 & 0.283 & 0.356 & 0.253 & 0.448 \\
\hline
\end{tabular}

Table 2. Mean and standard deviation of presence scores for participants whose fear was more than 50, d.f. 6 , '**' indicates significant differences.

\begin{tabular}{|l|l|l|l|l|}
\hline & Mean & P1 & P2 & P3 \\
\hline OST & $3.08 \pm 3.64$ & $1.38 \pm 2.07$ & $6.88 \pm 3.48$ & $1.00 \pm 1.51$ \\
\hline VST & $2.52 \pm 3.00$ & $1.36 \pm 3.26$ & $5.27 \pm 3.23$ & $0.91 \pm 1.58$ \\
\hline $\mathrm{t}$ & & 0.01 & 1.03 & 0.13 \\
\hline $\mathrm{p}$ & & 0.990 & 0.316 & 0.901 \\
\hline
\end{tabular}

Table 3. Mean and standard deviation of anxiety scores, d.f. 17, '**' indicates significant differences. 


\begin{tabular}{|l|l|l|l|}
\hline & $\mathrm{P} 1$ & $\mathrm{P} 2$ & $\mathrm{P} 3$ \\
\hline OST & $3.00 \pm 2.65$ & $9.33 \pm 1.16$ & $2.67 \pm 1.16$ \\
\hline VST & $2.75 \pm 1.50$ & $8.25 \pm 0.96$ & $1.75 \pm 2.06$ \\
\hline $\mathrm{t}$ & 0.16 & 1.36 & 0.68 \\
\hline $\mathrm{p}$ & 0.879 & 0.231 & 0.525 \\
\hline
\end{tabular}

4)

\begin{tabular}{|l|l|l|}
\hline $\mathrm{P} 1$ & $\mathrm{P} 2$ & $\mathrm{P} 3$ \\
\hline $0.40 \pm 0.89$ & $5.40 \pm 3.65$ & $0.00 \pm 0.00$ \\
\hline $0.57 \pm 1.51$ & $3.57 \pm 2.76$ & $0.43 \pm 1.13$ \\
\hline-0.23 & 0.99 & -0.83 \\
\hline 0.826 & 0.344 & 0.424 \\
\hline
\end{tabular}

5)

Table 4. Mean and standard deviation of anxiety scores for participants who had more fear, d.f. 5.

Table 5. Mean and standard deviation of anxiety scores for participants who had less fear, d.f. 10, '**', indicates significant differences.

\begin{tabular}{|l|l|l|}
\hline & $\mathrm{P} 2$ & $\mathrm{P} 3$ \\
\hline Mean \pm standard deviation & $5.27 \pm 3.23$ & $0.91 \pm 1.58$ \\
\hline $\mathrm{t}$ & $-4.80^{* *}$ & 2.19 \\
\hline $\mathrm{p}$ & $<0.001 * *$ & 0.053 \\
\hline
\end{tabular}

6)

\begin{tabular}{|l|l|}
\hline $\mathrm{P} 2$ & $\mathrm{P} 3$ \\
\hline $6.88 \pm 3.48$ & $1.00 \pm 1.51$ \\
\hline$-5.40 * *$ & 0.50 \\
\hline $0.001 * *$ & 0.633 \\
\hline
\end{tabular}

7)

Table 6. Comparing the initial level of anxiety with the level of anxiety in steps 2-3 using the VST. P1, $1.36 \pm 1.80$, d.f. 10 . Table 7 . Comparing the initial level of anxiety with the level of anxiety in steps $2-3$ using the OST. P1, 1.38 \pm 2.07 , d.f. 7, '**' indicates significant differences.

\begin{tabular}{|l|l|l|}
\hline & $\mathrm{P} 2$ & $\mathrm{P} 3$ \\
\hline Mean \pm standard deviation & $9.33 \pm 1.16$ & $2.67 \pm 1.16$ \\
\hline $\mathrm{t}$ & $-7.18^{* *}$ & 0.50 \\
\hline $\mathrm{p}$ & $0.019^{* *}$ & 0.893 \\
\hline
\end{tabular}

8)

\begin{tabular}{|l|l|}
\hline $\mathrm{P} 2$ & $\mathrm{P} 3$ \\
\hline $8.25 \pm 0.96$ & $1.75 \pm 2.06$ \\
\hline$-4.62 * *$ & 2.45 \\
\hline $0.019 * *$ & 0.092 \\
\hline
\end{tabular}

9)

Table 8. Comparing the initial level of anxiety with the level of anxiety in steps 2-3 using the OST and

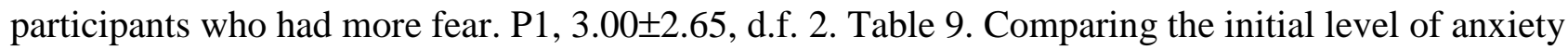
with the level of anxiety in steps 2-3 using the VST and participants who had more fear. P1, $2.75 \pm 1.50$, d.f. 3 , ' $* *$ ' indicates significant differences.

\begin{tabular}{|l|l|l|}
\hline & $\mathrm{P} 2$ & $\mathrm{P} 3$ \\
\hline Mean \pm standard deviation & $5.40 \pm 3.65$ & $0.00 \pm 0.00$ \\
\hline $\mathrm{t}$ & $-3.16^{* *}$ & 1.00 \\
\hline $\mathrm{p}$ & $0.034^{* *}$ & 0.374 \\
\hline
\end{tabular}

10)

\begin{tabular}{|l|l|}
\hline $\mathrm{P} 2$ & $\mathrm{P} 3$ \\
\hline $3.57 \pm 2.76$ & $0.43 \pm 1.13$ \\
\hline$-3.07 * *$ & 1.00 \\
\hline $0.02 * *$ & 0.356 \\
\hline
\end{tabular}

11)

Table 10. Comparing the initial level of anxiety with the level of anxiety in steps 2-3 using the OST and participants who had less fear. P1, $0.40 \pm 0.89$, d.f. 4. Table 11. Comparing the initial level of anxiety with the level of anxiety in steps 2-3 using the VST and participants who had less fear. P1, $0.57 \pm 1.51$, d.f. $6{ }^{\text {' }} * *$ ' indicates significant differences. 


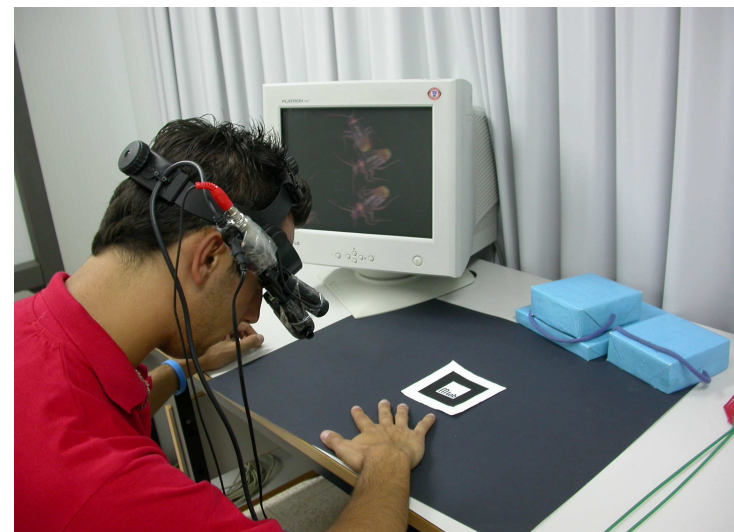

a)

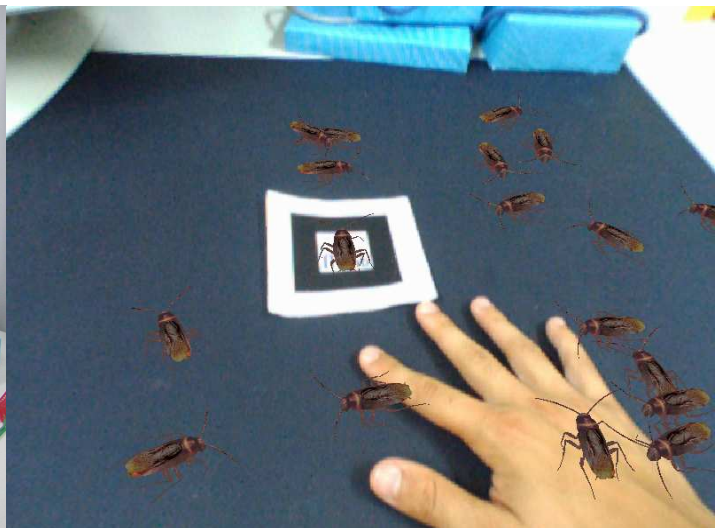

b)

Figure 3. A participant lets several cockroaches approach and cross over his hand. a) Participant b) Participant's view

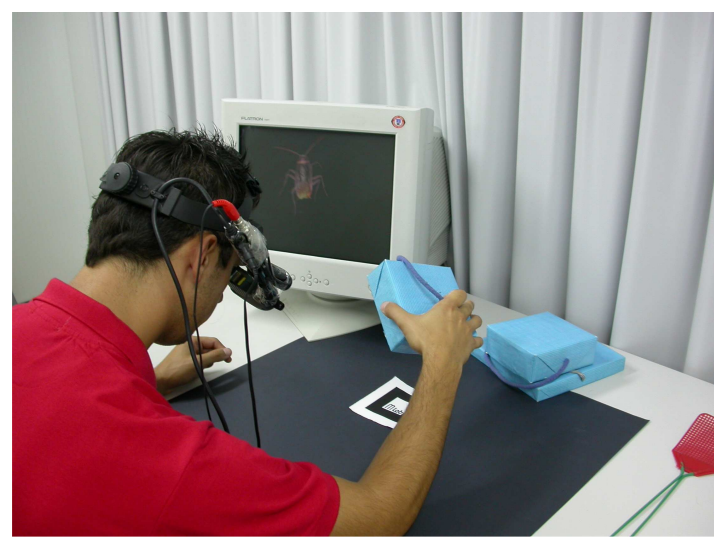

a)

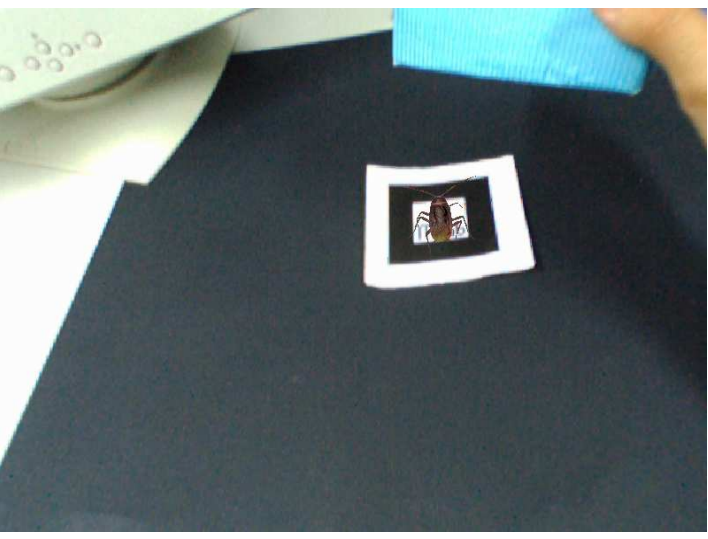

b)

Figure 4. A participant is searching for hidden animals. a) Participant b) Participant's view

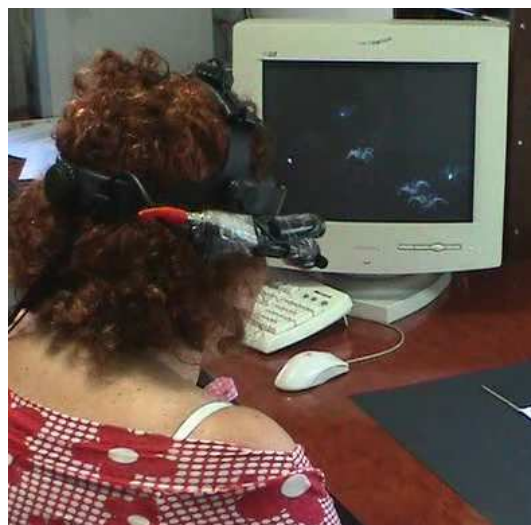

a)

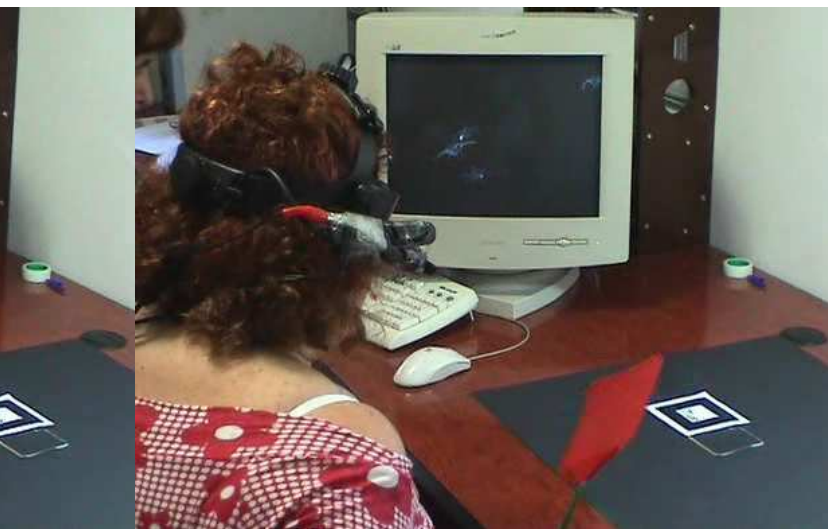

b)

Figure 5. a) A participant lets several spiders approach. b) A participant is killing spiders using the flyswatter 


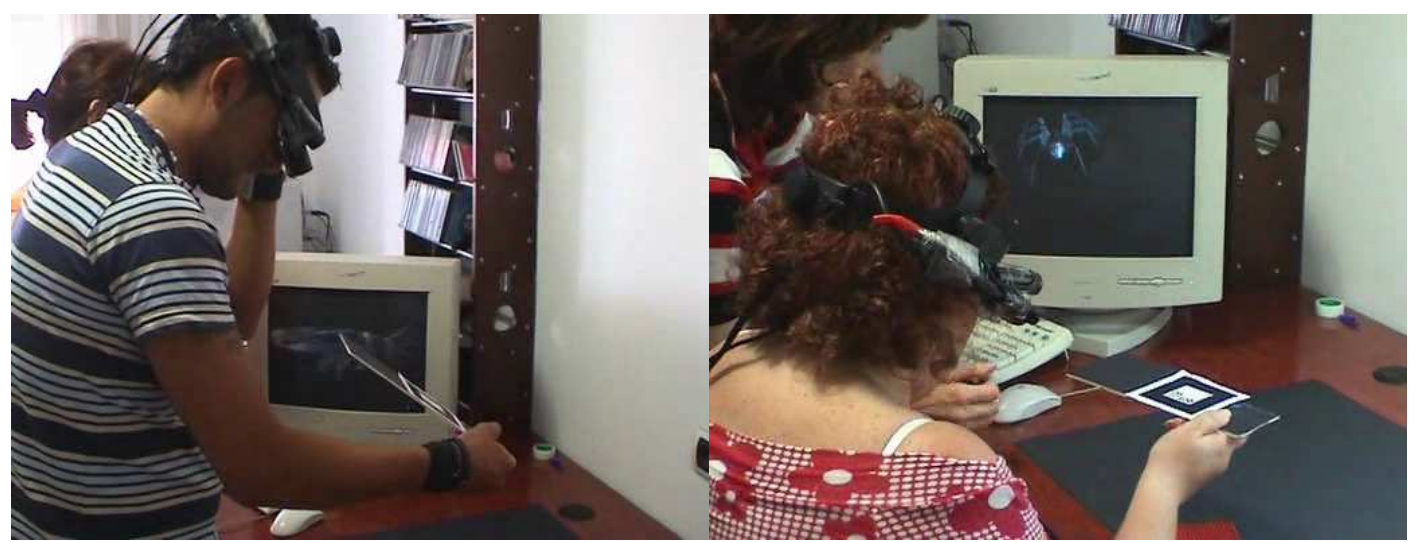

a)

b)

Figure 6. Participants looking at one animal nearby

\section{Conclusions}

We have presented the first OST AR system for the treatment of phobia to small animals. In this work, we have carried out a study with 24 non-phobic participants, and we have compared the OST and the VST systems. The results of the study indicate that, if all the participants are analyzed, the VST system induces greater sense of presence than the OST system and the order of exposure did not change the results. If the participants who had more fear are analyzed, the two systems induce a similar sense of presence. For the anxiety level, the two systems produced similar and significant anxiety during the experiment. The main drawback of this study is the number of participants in each group, especially in the subgroup of participants who had more fear, which had only seven participants. A new study with a larger sample would be required to be able to extract more statistically significant conclusions. Another future study is to run this experiment with a negative experimental control group to include non-frightening objects or animals in the testing system. The OST system can be improved in several ways that could reduce its differences with the VST system. First, the system uses a monocular HMD. This implies that the user does not see the same image with both eyes or that he/she has to close the left eye in order not to have two different images. This limits the generalization of this study. A binocular HMD would improve the current version and could contribute to a more realistic system. The binocular version will be stereoscopic because the animals will be placed with a slight displacement to each eye. The performance advantages associated with using either stereoscopic or monoscopic displays have already been explored (Nash et al., 2000), but usually using video seethrough HMD. Thus, a new study that explore the performance advantages related to monoscopic (using a binocular HMD and closing the eye without the microdisplay) versus stereoscopic optical seethrough HMDs and their comparison with video see-through HMDs could offer interesting contributions to the AR/VR fields. Second, the IR camera was manually fixed to the HMD. A more stable attachment could also improve the system. The differences between a VST and an OST visualization could also affect the results related to presence. According to Bimber \& Raskar (2005), OST visualization has some drawbacks with regard to VST visualization. One problem is that there are visual perception issues that are due to the constant image depth (the fixed focal-length problem). Since objects within the real environment and the image plane that is attached to the viewer's head are sensed at different depths, the eyes are forced to either continuously shift focus between the different depth levels or to perceive one depth level out of focus. Another problem is that OST devices require difficult (user- and session-dependent) calibration and require precise head-tracking to ensure a correct graphical overlay. Also, conventional optical see-through devices are incapable of providing consistent occlusion effects between real and virtual objects. This is due to the mirror beam splitters that reflect the light of the miniature displays, which interferes with the transmitted light of the illuminated real 
environment. To try to minimize this problem, in our OST system, we fixed a polarized material in order to reduce the influence of the exterior light.

Finally, the VST AR system has already been successfully used for treatment with real patients. Since the OST system induces a similar sense of presence for the group of participants who had more fear and also produced a similar and significant level of anxiety for all the participants during the experiment, OST could also be used for treatment with real patients. Future studies would help to analyze if there are any differences in treatment using the two systems.

\section{Acknowledgements:}

We would like to thank:

- Cristina Botella and Rosa Baños for their help.

- LabHuman for letting us use the material for developing and testing the systems.

\section{References}

Azuma, R. \& G. Bishop., G. (1994). Improving Static and Dynamic Registration in an Optical Seethrough HMD, Proceedings of 21st Annual Conference on Computer Graphics and Interactive techniques (SIGGRAPH '94), pp. 197-204

Bimber, O., Raskar, R., Spatial Augmented Reality. Merging Real and Virtual Worlds, Ed. A. K. Peters, 2005

Botella , C., Baños, R., Quero, S., Perpiñá, C. \& Fabregat, S. (2004). TelePsychology and Self-Help: The treatment of phobias using the Internet, Cybertherapy, CD-ROM.

Botella, C., Juan, M.C., Baños, R.M., Alcañiz, M., Guillen, V. \& Rey, B. (2005). Mixing realities? An Application of Augmented Reality for the Treatment of Cockroach phobia. Cyberpsychology \& Behavior, 8: 162-171.

Carlin, A., Hoffman, H. \& Weghorst, S. (1997) Virtual reality and tactile augmentation in the treatment of spider phobia: a case study, Behaviour Research and Therapy, 35(2): 153-158.

Fuhrmann, A., Schmalstieg, D., Purgathofer, W. (1999). Fast Calibration for Augmented Reality, ACM Virtual Reality Software \& Technology '99, pp. 166-167

García-Palacios, A., Hoffman, H. G., Carlin, A., Furness, T., \& Botella, C. (2002). Virtual Reality in the treatment of spider phobia: A controlled study. Behaviour Research and Therapy, 9: 983-993.

Genc, Y., Tuceryan, M., Navab, N. (2002) Practical solutions for calibration of optical see-through devices, pp. 169- 175

Grasset, R., Decoret, X., Gascuel, J. D. (2001) Augmented Reality Collaborative Environment: Calibration and Interactive Science Editing, Third Virtual Reality International Conference, http://artis.imag.fr/Publications/2001/GDG01/

Hoffman, H.G., Garcia-Palacios, A., Carlin, A., Furnes III, T. A., Botella, C. (2003). Interfaces that heal: Coupling real and virtual objects to treat spider phobia, International Journal of HumanComputer Interaction, 16(2): 283-300.

Ivanovic, A. and Plate, J. (2002) An augmented reality system on the construction site for different users, Virtual Environment on a PC Cluster Workshop, pp. 77-84

Juan, M.C., Alcañiz, M., Monserrat, C., Botella, C., Baños, R. \& Guerrero, B. (2005). Using Augmented Reality to treat phobias, IEEE Computer Graphics and Applications, 25(6): 31-37.

Juan, M.C., Joele, D., Baños, R., Botella, C., Alcañiz, M., van der Mast, Ch. (2006a) A Markerless Augmented Reality System for the treatment of phobia to small animals, The 9th International Workshop on Presence, pp. 71-74

Juan, M.C., Baños, R., Botella, C., Pérez, D., Alcañiz, M. \& Monserrat, C. (2006b). An Augmented Reality System for acrophobia: The sense of presence using immersive photography, Presence: Teleoperators \& Virtual Environments, 15: 393-402. 
Kato, H., \& Billinghurst, M. (1999). Marker tracking and HMD calibration for a video-based augmented reality conferencing system. IEEE and ACM International Workshop on Augmented Reality, pp. 85-94.

Leebmann, J. (2006) Application of an augmented reality system for disaster relief. International Archives of the Photogrammetry, Remote Sensing and Spatial Information Sciences, Vol. XXXIV-5/W10, http://www.photogrammetry.ethz.ch/tarasp_workshop/ papers/leebmann.pdf

Nash, E. B., Edwards, G. W., Thompson, J. A., Barfield, W. (2000) A review of presence and performance in Virtual Environments, International Journal of Human-Computer Interaction, 12(1): $1-41$

Owen, B.C., Zhou, Ji, Tang, A., Xiao, F. (2004) Display-Relative Calibration for Optical See-Through Head-Mounted Displays, Third IEEE and ACM International Symposium on Mixed and Augmented Reality (ISMAR'04), pp. 70-78

Özbek, C., Giesler, B., \& Dillmann, R. (2004) Jedi Training: Playful Evaluation of Head-Mounted Augmented Reality Display Systems, SPIE Conference Medical Imaging, 5291: 454-463

Renaud, P., Bouchard, S., \& Proulx, R. (2002). Behavioral avoidance dynamics in the presence of a virtual spider, IEEE Trans. on Information Technology in Biomedicine, 6(3): 235-243.

Schwald, B.; Laval, B (2003) An augmented reality system for training and assistance to maintenance in the industrial context, International Conference in Central Europe on Computer Graphics, Visualization and Computer Vision, pp.425-432

Slater, M., Usoh, M., \& Steed, A. (1994). Depth of presence in virtual environments. Presence: Teleoperators and Virtual Environments, 3: 130-144.

Szymanski, J. \& O'Donohue, W. (1995). Fear of spiders questionnaire. Journal of Behavior Therapy and Experimental, Psychiatry, 26(1): 31-34. 